\title{
Research on the Device Design for Calculation Distribution Line Loss Based on Dynamic Three-phase Unbalance Degree
}

\author{
WEI Meifang ${ }^{1}$, Yang Jing ${ }^{1}$, Zeng Xuhua ${ }^{1}$, Wu Haiyan ${ }^{1}$, Huang Di $^{1}$ \\ ${ }^{1}$ The Training Center of Hunan Electric Power Co, Ltd; China
}

\begin{abstract}
For the power distribution network line loss calculation, there are some problems, such as backward method, long calculation period, large workload and poor real-time performance. To this end, this paper proposes a real-time calculation method for distribution line loss based on dynamic three-phase unbalance, and a hardware device system with unbalanced phase sequence identification function, three-phase unbalance detection function and line loss real-time calculation function has been developed. The system uses a master-slave design based on the principle of wireless transmission. The slave collects standard three-phase current signals and transmits them to the host through wireless transmission. The host is installed on the low voltage side of the transformer in the transformer region, and the current signal of the transformer region is collected in real time. Unbalanced phase sequence identification, unbalance calculation, and line loss calculation based on dynamic unbalance are performed by comparing the current signals collected by the master and the slave. The above algorithm implementation process can be displayed online in real time. In addition to the core data processing and waveform display functions, the device also includes a battery power supply and management system, a signal storage and transmission system, and temperature measurement, and overcurrent and overvoltage protection. The test results of the device show that the device has the characteristics of convenient carrying, fast detection of three-phase unbalance, and accurate calculation of line loss. The device can monitor the running status of the distribution network transformer region in real time.
\end{abstract}

\section{Introduction}

Line loss is an important indicator of the economic efficiency and management level of power supply companies. For this reason, more and more attention are paid on line loss. However, for the line loss calculation, there are some problems, such as backward method, long calculation period, large workload, etc. To improve the line loss calculation method, a real-time calculation method for distribution line loss based on dynamic threephase unbalance is proposed. Based on the above algorithm, a hardware device system which has the function to identify the unbalanced phase and can calculate the line loss on line is presented as well in this paper.

\section{Three-Phase-Unbalance Calculation}

Degree

About three-phase unbalance degree, there are many definitions, such three-phase voltage unbalance degree and three-phase current unbalance degree. In order to describe the three-phase unbalance accurately, threephase current unbalance degree is adopted in this paper, The expression is as the follow,

$$
\left\{\begin{array}{l}
\beta_{A}=\frac{I_{A}-I_{a v}}{I_{a v}} \times 100 \% \\
\beta_{B}=\frac{I_{B}-I_{a v}}{I_{a v}} \times 100 \% \\
\beta_{C}=\frac{I_{C}-I_{a v}}{I_{a v}} \times 100 \%
\end{array}\right.
$$

Where, $\beta_{A}, \beta_{B}, \beta_{C}$ are the current imbalances of phase $\mathrm{A}$, phase $\mathrm{B}$ and phase $\mathrm{C}$, respectively; $I_{A}, I_{B}, I_{C}$ are the root mean square values of load currents of phase $A$, phase $\mathrm{B}$ and phase C, respectively, (A); $I_{a v}$ is the average value of the RMS three phases current, (A).

From above function, we know that every phase of the distribution line has its own unbalance degree, that means the current unbalance of the three phase are quite different. But for the distribute network, the maximum three-phase current can cause severe harm. So, the highest of threephase current unbalance degree taken as the definition to represent unbalance level of the transformer section.

\section{Calculation of the Line Loss}

At present, RMS current method, average current method, and maximum current method are used to calculate line loss. ${ }^{[1-2]}$. However, these methods have the high practicability under the condition that three-phase load 
distribution is relatively balance ${ }^{[3]}$. If this method is applied to unbalanced transformer section which may result in for the reason that these method didn't take the unbalanced current into consider. ${ }^{[4-5]}$.

To solve this problem, the current imbalance is considered when calculating the line loss of the transformer part.

The expression is as the follow,

$$
I_{\phi}=\left(\beta_{\phi}+1\right) I_{a v}
$$

Where, $I_{\phi}$ is the RMS value of each phase current, (A); $\beta_{\phi}$ is the imbalance degree of each phase; $I_{a v}$ is the average value of the RMS value of the three-phase current, (A).

The three-phase unbalance will cause a change in the three-phase current. According to formula (2), deriving the expression of the RMS value of the three-phase current under unbalanced state. The formula is as follows:

$$
I_{\text {mav }}=\sqrt{\frac{\left[\left(\beta_{A}+1\right)^{2}+\left(\beta_{B}+1\right)^{2}+\left(\beta_{C}+1\right)^{2}\right]}{3}} I_{a v}
$$

(3) Where,

$I_{\text {mav }}$ is the RMS value of the three-phase current under unbalanced state, (A), $\beta_{A}, \beta_{B}, \beta_{C}$ are the imbalance degree of each phase; $I_{a v}$ is the average value of the RMS value of the three-phase current, (A).

The three-phase unbalance not only causes the threephase line current to change, but also causes the neutral line current to change. The expression is as the follow,

$$
I_{N=}\left[\left(\beta_{A}+1\right)+\left(\beta_{B}+1\right)\left(-\frac{1}{2}-j \frac{\sqrt{3}}{2}\right)+\left(\beta_{C}+1\right)\left(-\frac{1}{2}+j \frac{\sqrt{3}}{2}\right)\right] I_{a v}
$$

Where, $I_{N}$ is the neutral current under unbalanced state, (A).

According to the formula (3) (4), the rate of change of current introduced into the unbalanced state can be obtained.

$$
K_{I}=\frac{3 I_{\operatorname{mav}}+I_{N}}{3 I_{a v}}
$$

Where, $K_{I}$ is the rate of change of current to introduce an unbalanced state.

This paper uses an improved equivalent resistance method for the calculation of line equivalent resistance. Some assumptions are taken:(1) The load distribution is consistent with the rated capacity of the distribution transformer, and the load rates of the nodes of the segmentation line are the same; (2) Ignoring the voltage difference of each node.

Based on the above assumptions, the equivalent resistance expression can be obtained as:

$$
R_{e l}=\frac{\sum_{i=1}^{n}\left(A_{P i}^{2}+A_{Q i}^{2}\right) R_{i}}{A_{P}^{2}+A_{Q}^{2}}
$$

Where, $R_{e l}$ is the equivalent resistance, $(\Omega) ; A_{P i}$ is the active power of the $\mathrm{i}$-th line resident user, $(\mathrm{kWh}) ; A_{Q i}$ is the reactive power of the $\mathrm{i}$-th line resident user, $(\mathrm{kWh})$; $R_{i}$ is the resistance value of the $\mathrm{i}$-th line, $(\Omega) ; A_{P}$ is the total active power of the low-voltage outlet side of the transformer in the station area, $(\mathrm{kWh}) ; A_{Q}$ is the total reactive power of the low-voltage outlet side of the transformer in the station area, $(\mathrm{kWh}) ; \mathrm{n}$ is the total number of sections of the station line.

The line loss can be easily obtained based on the line voltage, current, and equivalent resistance. For a monthly, the line loss expression is:

$$
\Delta A=N I_{m a v}^{2} K^{2} R_{e l} T \times 10^{-3}
$$

Where, $\Delta A$ is line loss for a period of time, $(\mathrm{kWh}) ; N$ is the low-voltage side outlet structure coefficient of the distribution transformer, the single-phase two-wire system power supply takes 2 , the three-phase three-wire system power supply takes 3 , and the three-phase fourwire system power supply takes $3.5 ; I_{\text {mav }}$ is monthly average load current, (A); $\mathrm{K}$ is the shape coefficient of the load side of the distribution transformer; $R_{e l}$ is the line equivalent resistance, ( $\Omega$ ); $\mathrm{T}$ is the calculation period, taking the total number of hours in this period.

Merge formula (5) and formula (7) results in line losses in an unbalanced state.

$$
\Delta A=K_{I}^{2} N I_{\text {mav }}^{2} K^{2} R_{e l} T \times 10^{-3}
$$

Where, $\Delta A$ is line loss for a period of time, $(\mathrm{kWh})$; $K_{I}$ is the rate of change of current to introduce an unbalanced state

\section{System Design to Calculate the Line Loss}

The distribution line loss calculation device based on dynamic three-phase unbalance can identify phase sequence identification on the user side, dynamic imbalance calculation and line loss calculation based on dynamic unbalance. The implementation theory is as follows:

1) Phase sequence identification function: Phase sequence identification is realized by the principle of wireless transmission, the master-slave device design, and comparing the detection terminal signal collected by the master and the three-phase current signal collected by the slave. The theory of phase sequence identification is to compare the current signal of the detection end as a standard signal, and compare it with the current signal of the output side of the distributed distribution transformer at the same time.

2) Dynamic unbalance calculation function: The calculation method of dynamic unbalance degree, through the slave machine to collect the three-phase current signal of the transformer outlet side of the station, send it to the master, process the data through the stm 32 chip of the host, and calculate according to formula (1) Dynamic threephase current imbalance.

3) Line loss calculation function based on dynamic unbalance: The master's stm 32 chip reprocesses the calculated dynamic imbalance data. The current and voltage on the outlet side of the transformer measured by the slave, the structural coefficient of the transformer outlet side input in advance, the shape curve of the load curve, the equivalent resistance of the line, and the number of hours in the calculation period are input in advance. The line loss value is calculated by formula (8). 


\section{Hardware Design}

The device is a master-slave design based on wireless communication, and is composed of a master and a slave. The master is installed on the user side to collect the standard current signal of the user side. The slave machine collects the three-phase current signal at the exit side of the transformer. The master and slave data are integrated and processed through the wireless communication module to identify the phase sequence on the user side, and calculate the dynamic imbalance and the line loss value based on the dynamic imbalance.

The master/slave is composed of a current collecting module, a signal conditioning circuit, a wireless communication module, a power module, a voltage stabilizing circuit, a display screen, a data storage module, a temperature measuring module, and a data processing unit. The working process is: 1) The current at the outlet side of the transformer is collected by the current-voltage converter for measurement and converted into a voltage signal that is easy to change and transfer. 2) Conditioning the signal collected by the acquisition module into a signal that can be input to the main control chip. 3) The collected signal is processed in the microcontroller. And encode the data into a serial signal. 4) The serial port signal is transmitted to the host through the wireless serial port module. 5) The host collects standard signals. The standard signals and the three-phase current signals from the slave are processed in the microcontroller. The phase sequence discrimination result and the imbalance degree and the line loss value are obtained. 6) STM32 send these calculation results to the display. The hardware structure of the slave is shown in Figure 1

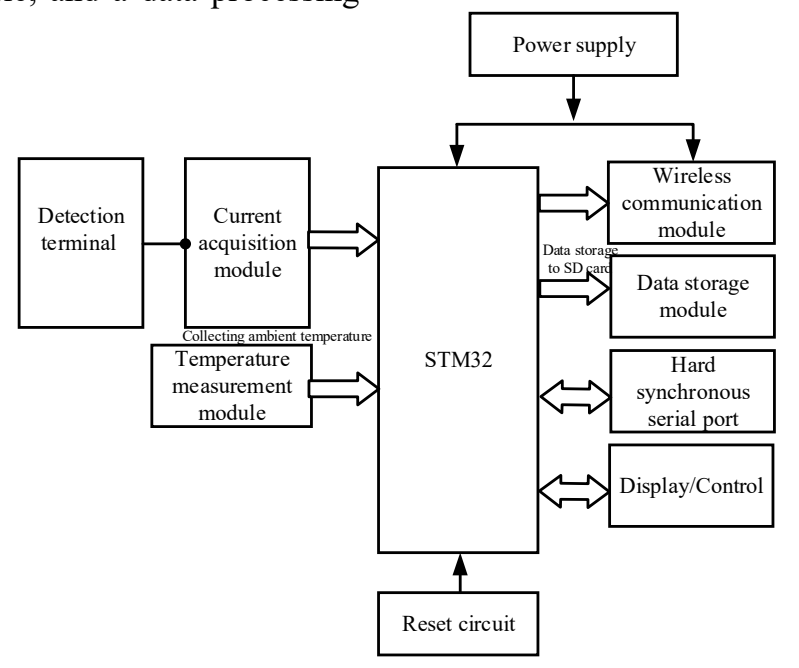

Fig. 1. Schematic diagram of the hardware structure of the master/slave

The communication between the master and the slave is realized through the wireless serial port module and an in-line DB9 serial port. In the phase sequence identification of the transformer area, it is necessary to compare the current signal collected by the master and the three-phase current signal collected by the slave. Calculate the phase of the four signals at the same time and compare them. When the phase difference between the standard signal and a phase of the three-phase current signal is less than $5^{\circ}$, the discrimination result is obtained. Therefore, it is not only necessary to remotely transmit current, temperature and other data through the wireless serial port module, but also to use a serial communication to synchronize the clock. Because only the phase of the current measured at the same clock can be used to determine the phase sequence of the zone. Therefore, the serial port communication should be used to synchronize the sampling clocks of the master and the slave before the test to ensure that the master slaves simultaneously sample. The communication is shown in Figure 2.

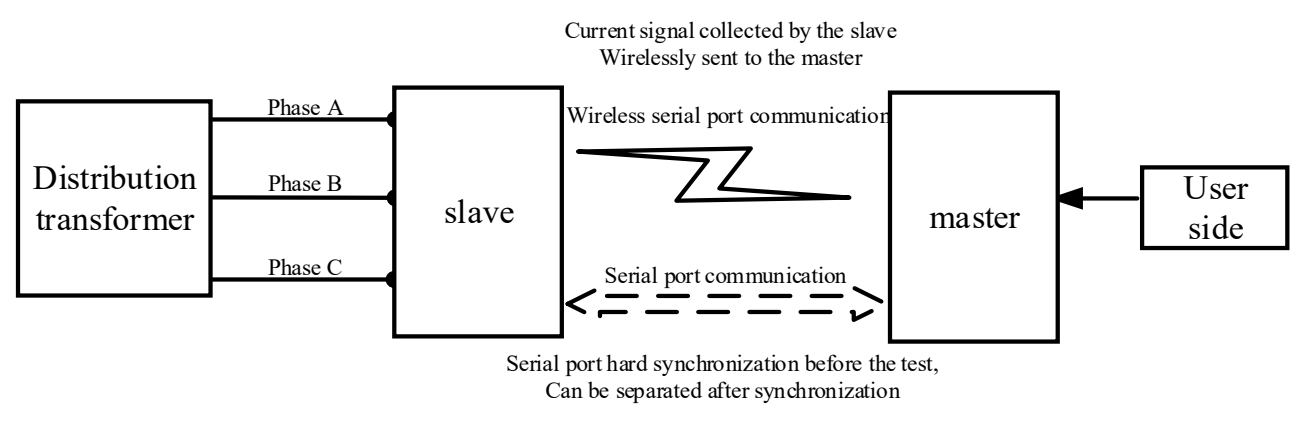

Fig. 2.Schematic diagram of host slave communication 


\section{Software Design}

The program flow chart is shown in Figure 3. After installation and power on the device, configure the sampling delay time and other parameters on the serial screen of the host, and start collecting current data after delaying the specified time. According to the current imbalance and line loss calculation algorithm in the program, the collected data is used to calculate the dynamic unbalance and the real-time line loss, and the phase sequence discrimination result is obtained according to the zero-crossing detection method in the program. The data acquisition calculation is shown in the display. The acquired and calculated data can be selectively displayed on the display and stored in the SD card.

\section{Conclusion}

To solve the problems of line loss calculation in the transformer section, this paper proposes a real-time calculation method of line loss based on dynamic threephase unbalance, and develops a set of distribution line loss calculation device with unbalanced phase sequence identification function, three-phase unbalance detection function and line loss real-time calculation function. The conclusions are summarized as follows:

1) This paper studies the calculation method of unbalance of three-phase dynamic load and line loss, and USES the improved equivalent resistance method to calculate line loss. This method is more reasonable and accurate for the calculation of line loss.

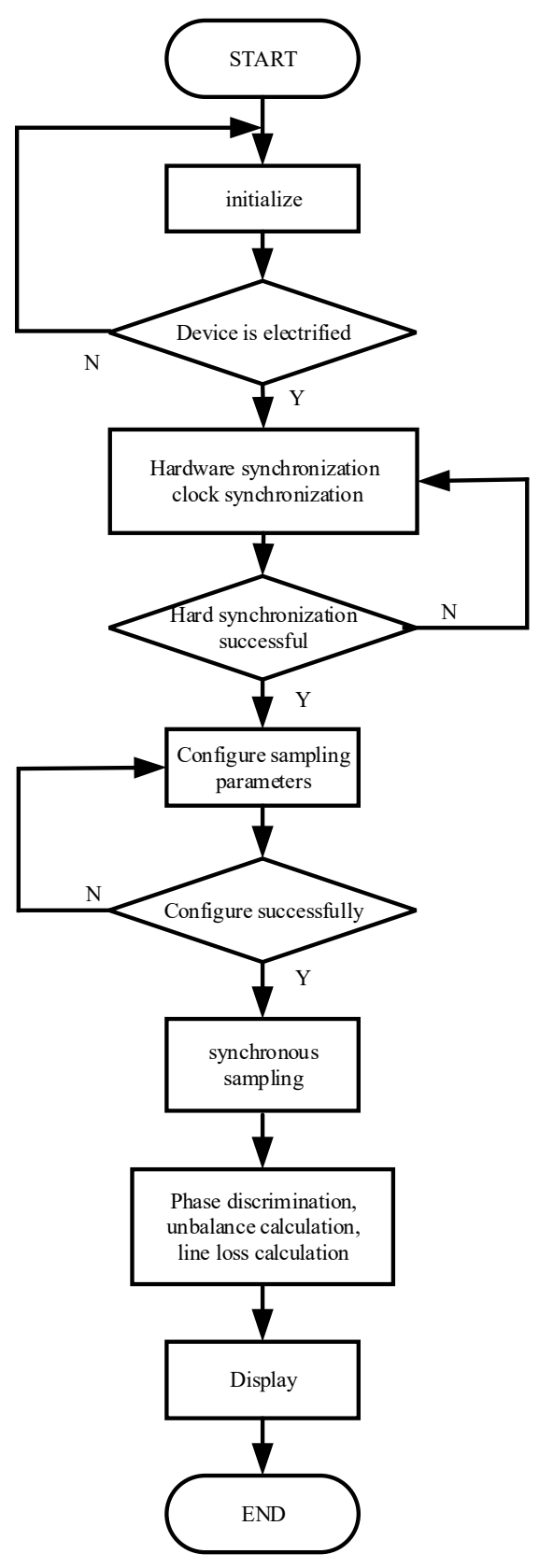

Fig. 3. Program flow chart of distribution line loss calculation device based on dynamic three-phase unbalance

2) The device is based on the master-slave system design method based on the wireless transmission principle, and the core algorithm include improved equivalent resistance method, the line loss calculation method considering the dynamic load three-phase unbalance degree and the zero-crossing phase sequence detection method. Based on the above software and hardware designs, this set of distribution line loss calculation device based on dynamic three-phase unbalance is developed.

3) This device is fast and accurate. The device is lightweight and easy to use. The put into use of the device can solve the problems such as line loss calculation method is backward, the calculation period is long, the workload is large, and the real-time performance is poor. 


\section{References}

1. ZHANG Qiaoxia, CHANG Gang. A Study of Line Loss Calculation Method and Its Application in Distribution Network [J]. China Rural Water and Hydropower, 2015(5):180-184.

2. ZU Kaitao, LIN Zhuoqiong. Line Loss Fine Calculation and Loss-reducing Analysis [J]. Shaanxi Electric Power, 2016, 44(1):60-64.

3. HUANG Xiaoyun, OUYANG Weinian, WU Shuhong. Theoretical Line Loss Calculation Method for Smart Distribution Network Based on Quasi Real Time Data [J]. Automation and instrumentation, 2016(4):130131.

4. SHU Qiqi, XIAO Jianhong, REN Weimeng. Research on Distribution Line Loss Analysis Method in Power System [J] Automation and instrumentation, 2018(3).

5. AN Wenpeng, WANG Bing, ZHOU Ning. Modeling of LV Distribution Network Based on Metering Data [J]. Power System Technology, 2015, 39(11):31413146.

6. ENG Hua, CHEN Honglin, LI Xinran. A Method for Synchronous Line Loss Statistics of Distribution Network Based on Load or Electricity Consumption Forecasting [J]. Power System Protection and Control, 2016, 44(18):108-114.

7. ANG Zhiwei. Design and Implementation of Three Phase Unbalanced Monitoring Device for Low
Voltage Distribution Network[D]. Taiyuan University of Technology, 2015.

8. HANG Xiaoyi, LI Yuxuan, HE Jian. Application of the Hybrid Compensation Regulator in Three-phase Unbalanced Load of the Low-voltage Distribution Network [J]. Electrical Automation, 2017, 39(2):8891.

9. HANG Peng, LUO Jian, JI Chang. Three-phase Unbalanced Load Parameters Identification of Low Voltage Power Utilization Network [J]. Power System Protection and Control, 2015, 43(24):90-95.

10. hang Yitao, Wang Zhezhong Liu Liping, et al. Line Loss Prediction of $10 \mathrm{kV}$ Distribution Network Based on Grey Correlation Analysis and Improved Neural Network[J]. Power System Technology, 2019(4).

11. heng Zhiyu, Li Shidong, Ling Shuchang, et al. Calculation and Analysis of Line Loss in Medium and Low Voltage DC Distribution Network[J]. Electrical measurement and instrumentation, 2017, 56(3).

12. Lu Bei, Zhang Hongyan, Sun Hui, et al. Low-voltage grid line loss calculation and management system based on browser/server architecture[J]. Power System Technology, 2010(2):211-214.

13. Li Ruichao,Wang Song,Huang Taigui, et al. Key Technology of Early Warning Analysis of Power Line Loss and Stealing Based on Big Data Platform[J]. Power System Protection and Control, 2018 